\title{
SPATIAL ADJACENCY ANALYSIS OF CITYGML BUILDINGS VIA 3D TOPOLOGICAL DATA STRUCTURE
}

\author{
Salleh, S. ${ }^{*}$, Ujang, U., Azri. S. and Choon, T.L. \\ Geoinformation, Faculty of Built Environment and Surveying, Universiti Teknologi Malaysia (UTM), Johor, Malaysia - \\ syahiirahsalleh78@gmail.com, \{mduznir, suhaibah, tlchoon\}@utm.my
}

KEY WORDS: Adjacencies, 3D topology, CityGML, 3D city modelling

\begin{abstract}
:
Adjacencies between objects provides the most basic connectivity information of objects. This connectivity information provides support for more complex 3D spatial analysis such as 3D navigation, nearest neighbour and others. In 3D models, the connectivity information is maintained by building a comprehensive 3D topology. As the international standard for 3D city models, CityGML employs a simple XML links mechanism that references related entities to each other as a means of maintaining topological information. This method fulfils the purpose of relating connected entities but, it does not describe how the entities are related or in other words its adjacencies. In this study, a 3D topological data structure was utilised to preserve topological primitives and maintain connectivity information for CityGML datasets of buildings in LoD2. The adjacencies tested in this study were based on the topological links maintained by the Compact Abstract Cell Complexes 3D topological data structure. Four types of adjacencies were tested which are Point-to-Line, Line-to-Surface, Surface-to-Surface and Volume-to-Volume adjacency. As a result, all adjacencies were able to be executed for both datasets which consisted of two connected buildings and disjointed buildings. It was found that the ability of the 3D topological data structure to preserve topological primitives and build topological links supported the maintenance of connectivity information between buildings. The maintenance of connectivity information was also not limited to objects of the same dimension and could extend to connectivity between building elements in different dimensions.
\end{abstract}

\section{INTRODUCTION}

CityGML is the international standard for 3D city modelling which represents buildings and other city elements in 3D. It has been widely used in various applications (Keling et. al. 2017; Mohd et. al. 2017; Ujang et. al. 2018; Azri et. al. 2014). The three main components of CityGML are geometry, semantics and topology ( $\mathrm{Li}$ et al., 2016). The geometric component of CityGML handles the construction of objects using geometric primitives as specified by the GML3 geometric model classes (Kolbe, 2009). Another important component of CityGML which is the semantics handles the thematic representation of the objects such as buildings, water bodies, transportation and others (Biljecki et al., 2016). The topological component of CityGML is implemented using XML link mechanism or XLinks which references related objects in XML format as shown in Figure 1 (Gröger \& Plümer, 2012; Kolbe, 2009; Li et al., 2016).

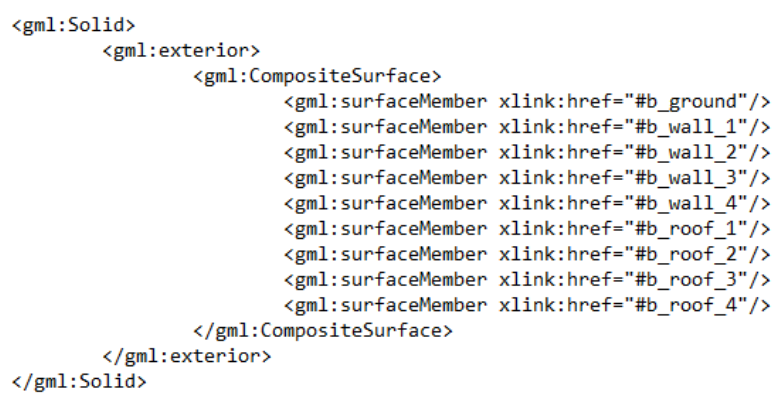

Figure 1. XML links mechanism in CityGML

Topology can be generally explained as the relative positions or characteristics of objects within a geometric space which remain unchanged regardless of any changes to the geometric space (Martinez-Llario et al., 2017; Worboys \& Duckham, 2004). There are three properties of topology which makes the topological representation of an object equally important to the geometric representation. Firstly, the topological representation of an object takes into account the distances between elements which make up the object without being constrained by the coordinate system (Carlsson, 2014). Next, the topological properties of the object remain unchanged regardless of any deformation to the object (Carlsson, 2014). Finally, the topological representation of an object offers a compressed version of the object while still preserving qualitative features such as relationships (Carlsson, 2014). The topological and geometric properties of an object both corresponds to the primitives that make up the object such as nodes, edges and faces hence allowing the topological properties to maintain information of adjacencies between objects or elements of the object (Bruzzone \& De Floriani, 1991).

The XLinks mechanism as the topological component of CityGML supports a simple topology which is unable to directly retrieve topological relationships (Rook et al., 2016). The simple topology does not provide support for topological analysis such as 3D adjacencies and nearest neighbour (Ujang et al., 2019). Apart from that, the XLinks mechanism requires the common surfaces of related objects to be explicitly stored and represented as individual geometries ( $\mathrm{Li}$ et al., 2016). This is caused by the absence of support for topological primitives and inability to maintain 3D topology (Boguslawski et al., 2011; Li et al., 2016). Consequently, relationships between topological primitives of different dimensions are also not maintained (Ghawana \& Zlatanova, 2012). As an effort to preserve topological properties and maintain topological relationships, a 3D topological data structure can be implemented. 
This paper attempted to perform analysis on adjacencies between buildings and building elements with the implementation of a 3D topological data structure. The methodology for executing the adjacency analysis is detailed in Section 2. Next, the analysis of adjacencies and results are discussed in Section 3. Finally, the conclusion is presented in Section 4.

\section{METHODOLOGY}

In order to maintain topological information, a 3D topological data structure was implemented for the buildings datasets in LoD2 CityGML. This is crucial in further analysis such as 3D data quantization (i.e. Azri et. al. 2016; Azri et. al. 2017). The Compact Abstract Cell Complexes (CACC) 3D topological data structure was implemented where topological links are generated from the geometrical properties of the buildings. These links consist of alpha- $0\left(\alpha_{0}\right)$ links which connects vertices to form edges, alpha- $1\left(\alpha_{1}\right)$ links which connects edges to form surfaces, alpha-2 $\left(\alpha_{2}\right)$ links which connects surfaces to form volumes and alpha-3 $\left(\alpha_{3}\right)$ links which represents the connectivity between volumes (Ujang et al., 2014). An example of the CACC topological links are depicted in Figure 2.

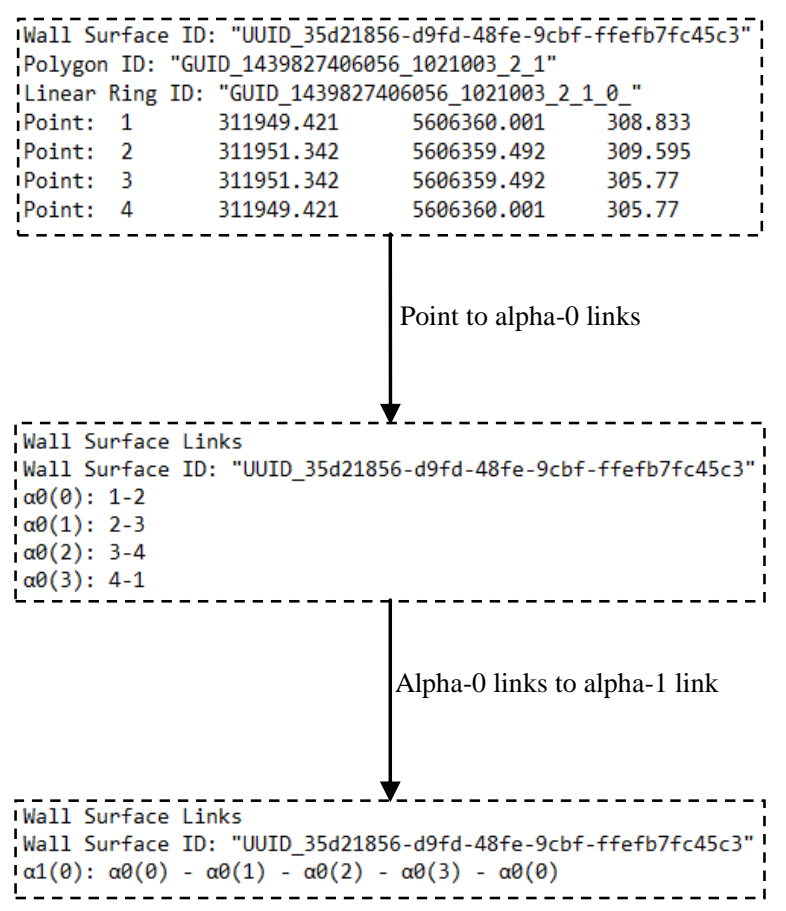

Figure 2. Generation of alpha-0 and alpha-1 links from 0D points

The topological links act as building blocks to construct links of a higher dimension then the previous link. For instance, 1D edges represented as $\alpha_{0}$ links are connected to form one $\alpha_{1}$ link that represents a $2 \mathrm{D}$ surface and in turn $\alpha_{1}$ links are connected to form one $\alpha_{2}$ link that represents a 3D volume. Reciprocally, the topological links can also be broken down to lower dimensions. This ensures the connectivity between elements of a building without being constrained to its dimensionality. The procedures to generate these topological links using the CACC 3D topological data structure is detailed in Salleh et al. (2018).
For this study, an adjacencies query function was developed within the program that generated the topological links. The adjacencies function of the program is shown in Figure 3.

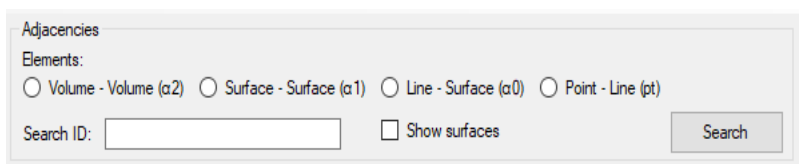

Figure 3. Adjacencies function interface

Four types of adjacencies are tested using the adjacency function which are Point-to-Line, Line-to-Surface, Surface-toSurface and Volume-to-Volume. Each adjacency requires user input of the search ID which corresponds to the object or topological link being queried. Each adjacency will be explained further in the subsequent sections.

The buildings in LoD2 CityGML used in this study consists of two datasets where dataset $\mathrm{A}$ is two connected buildings while dataset B is two disjointed buildings as shown in Figure 4.
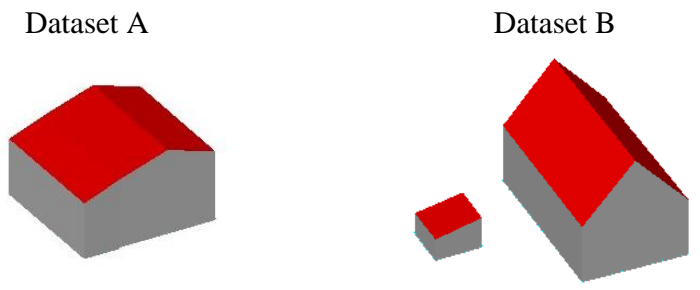

Figure 4. Building datasets in LoD2 CityGML

\section{ADJACENCY ANALYSIS AND RESULTS}

The primitive elements of the buildings are represented by topological primitives which consists of vertex (v), edge (e), face (f) and volume. The retrieval of adjacent elements by using the query function is elaborated and query results are illustrated in the subsequent sections. Each of the adjacency queries performed resulted in textual output which was also accompanied by visualisation of the results where the targeted geometries are coloured in red while adjacent geometries are coloured in yellow.

\subsection{Point-to-Line Adjacency}

The point-to-line adjacency returns all edges represented as alpha-0 links $\left(\alpha_{0}\right)$ that are adjacent to the vertex being queried $\left(v_{I D}\right)$. An alpha-0 link consists of two connected vertices where the prior vertex is known as the head ( $\left.v_{\text {hend }}\right)$ while the vertex ending the alpha-0 link is known as the tail $\left(v_{t a i l}\right)$. The set of adjacent edges to the queried point can be denoted as set $\mathrm{A}$ shown below;

$$
A=\left\{\alpha_{0}(i) \mid v_{I D} \in \alpha_{0}(i):\left(v_{I D}=v_{\text {head }_{\alpha}(i)}\right) \vee\left(v_{I D}=v_{\text {tail }_{\alpha_{0}(i)}}\right)\right\}
$$

The $\alpha_{0}(i)$ links adjacent to $v_{l D}$ are links such that $v_{l D}$ is an element of $\alpha_{0}(i)$ where $v_{I D}$ is equal to $v_{\text {hend }}$ or $v_{t a i l}$ of the $\alpha_{0}(i)$. Table 1 depicts some results of the point-to-line adjacency analysis for both datasets. 


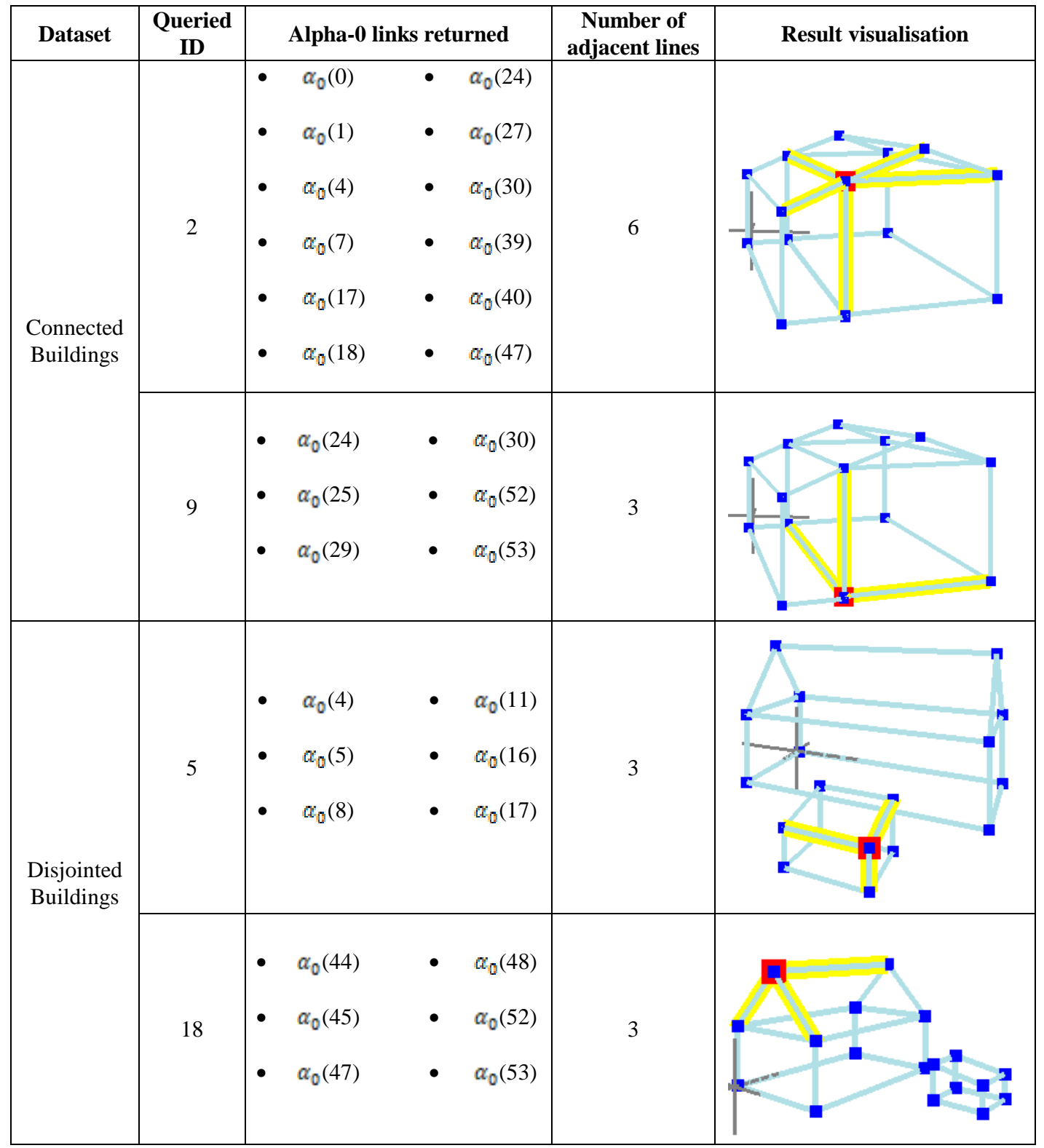

Table 1. Point-to-Line adjacency results

\subsection{Line-to-Surface Adjacency}

The line-to-surface adjacency retrieves all alpha-1 $\left(\alpha_{1}\right)$ links which represents surfaces adjacent to a queried line or alpha- 0 link $\left(\alpha_{0}\right)$. The surfaces represented by individual $\alpha_{1}$ links which are built up of $\alpha_{0}$ links are searched to retrieve the adjacent surfaces for the queried line. The adjacent surfaces can be denoted as set $\mathrm{A}$ as shown in the following equation (2);

$$
A=\left\{\alpha_{1}(i) \mid \alpha_{0}(I D) \in \alpha_{1}(i)\right\}
$$

The $\alpha_{1}$ links retrieved as adjacent surfaces are $\alpha_{1}$ links that contains the queried $\alpha_{0}$ link, $\alpha_{0}(I D)$ as an element of the retrieved $\alpha_{1}$ link. Table 2 illustrates examples of the line-tosurface adjacency analysis for both datasets. 


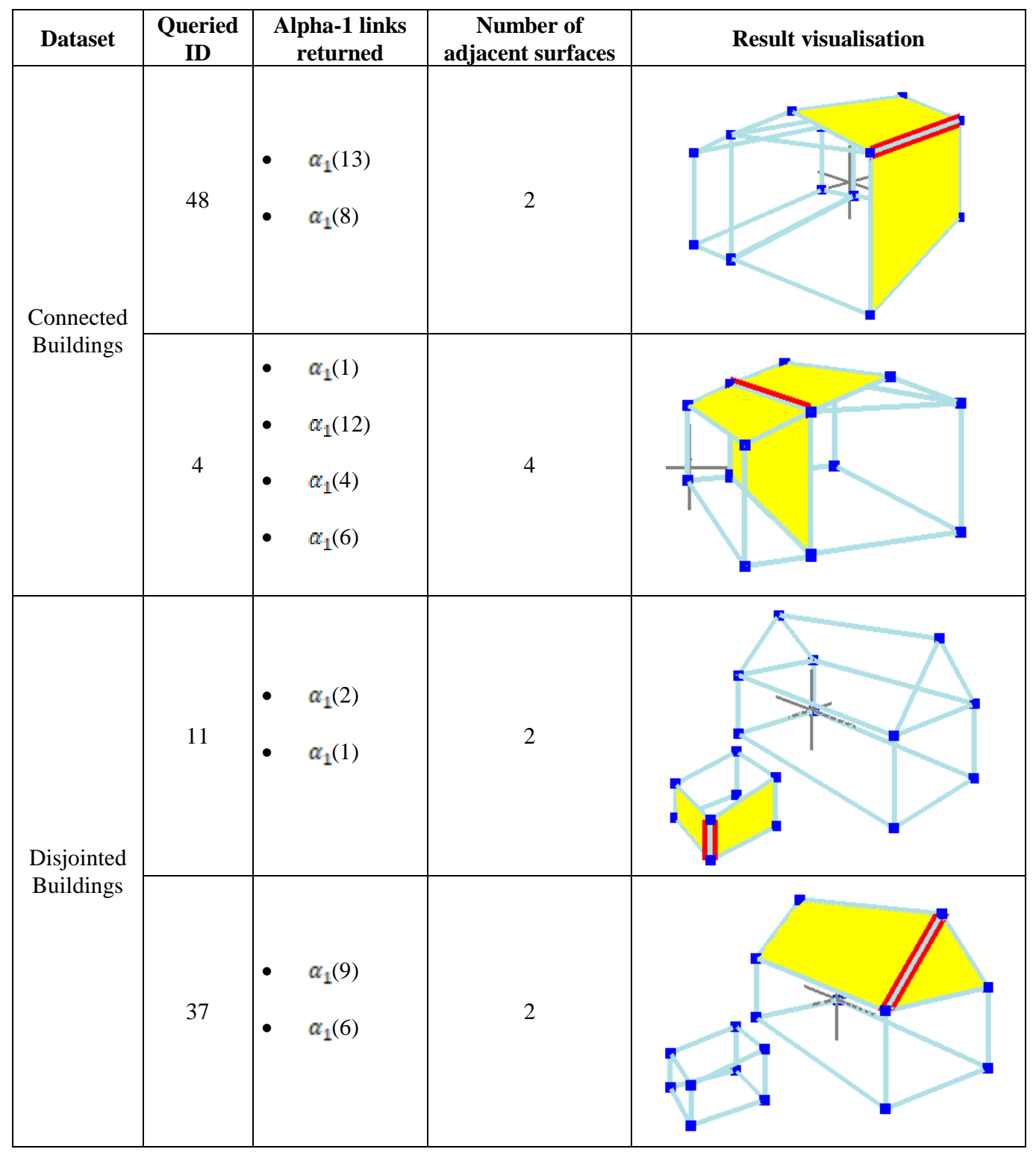

Table 2. Line-to-Surface adjacency results

\subsection{Surface-to-Surface Adjacency}

The surface-to-surface adjacency returns surfaces represented by alpha-1 $\left(\alpha_{1}\right)$ links which are adjacent to the surface being queried $\alpha_{1}(I D)$. Surfaces are found to be adjacent if a common line is shared between the surfaces making them traversable. The adjacent surfaces can be denoted as below;

$$
A=\left\{\alpha_{1}(i) \mid \alpha_{0}(i) \in \alpha_{1}(I D) \wedge \alpha_{0}(i) \in \alpha_{1}(i)\right\}
$$

Adjacent surfaces to the queried surface are expressed as set A which are composed of $\alpha_{1}$ links such that there is an $\alpha_{0}$ link which is an element of the queried $\alpha_{1}(I D)$ and is also an element of the adjacent surface $\alpha_{1}$. Table 3 describes a few examples of the surface-to-surface adjacency analysis conducted on both datasets. 


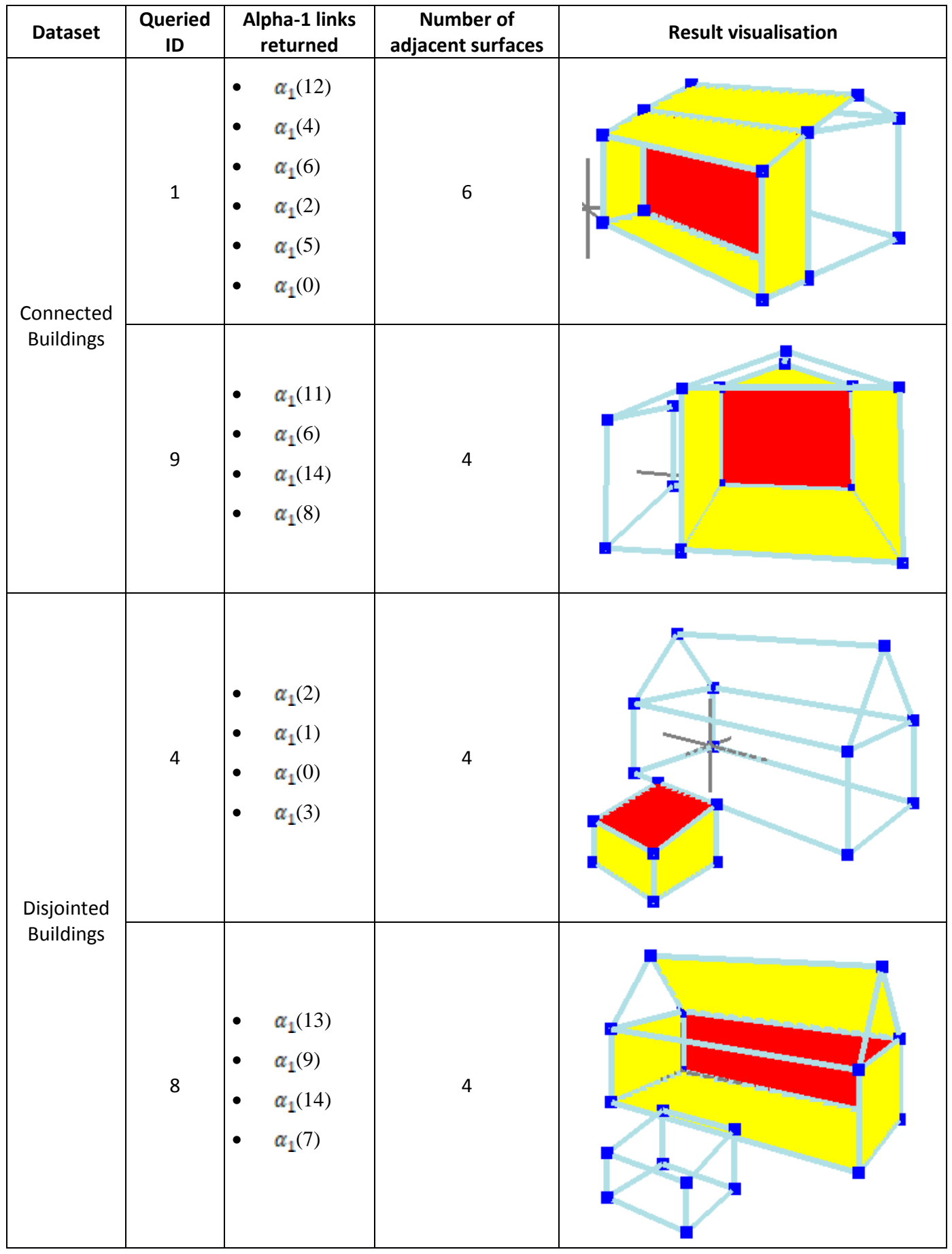

Table 3. Surface-to-Surface adjacency results

\subsection{Volume-to-Volume Adjacency}

The volume-to-volume adjacency returns volumes as alpha-2 links $\left(\alpha_{2}\right)$ where there exists topological connectivity between volumes represented by alpha-3 links $\left(\alpha_{3}\right)$ and the queried volume $\alpha_{2}(I D)$. The $\alpha_{2}$ links that are adjacent can be expressed as set A shown below;

$$
\begin{gathered}
A=\left\{\alpha_{2}(i) \mid \alpha_{2}(I D) \in \alpha_{3}(i) \cap \alpha_{2}(i) \in \alpha_{3}(i)\right\}, \\
\text { iff } \alpha_{3}(i) \neq \phi .
\end{gathered}
$$

The adjacent volumes to the queried volume is denoted as set $\mathrm{A}$ of $\alpha_{2}$ links such that the queried volume $\alpha_{2}(I D)$ is an element of the $\alpha_{a}$ intersects the adjacent volume $\alpha_{a}(i)$; if and only if $\alpha_{3}(i)$ is non-empty. Table 4 depicts some examples of the volume-to-volume adjacency analysis performed on both datasets. 


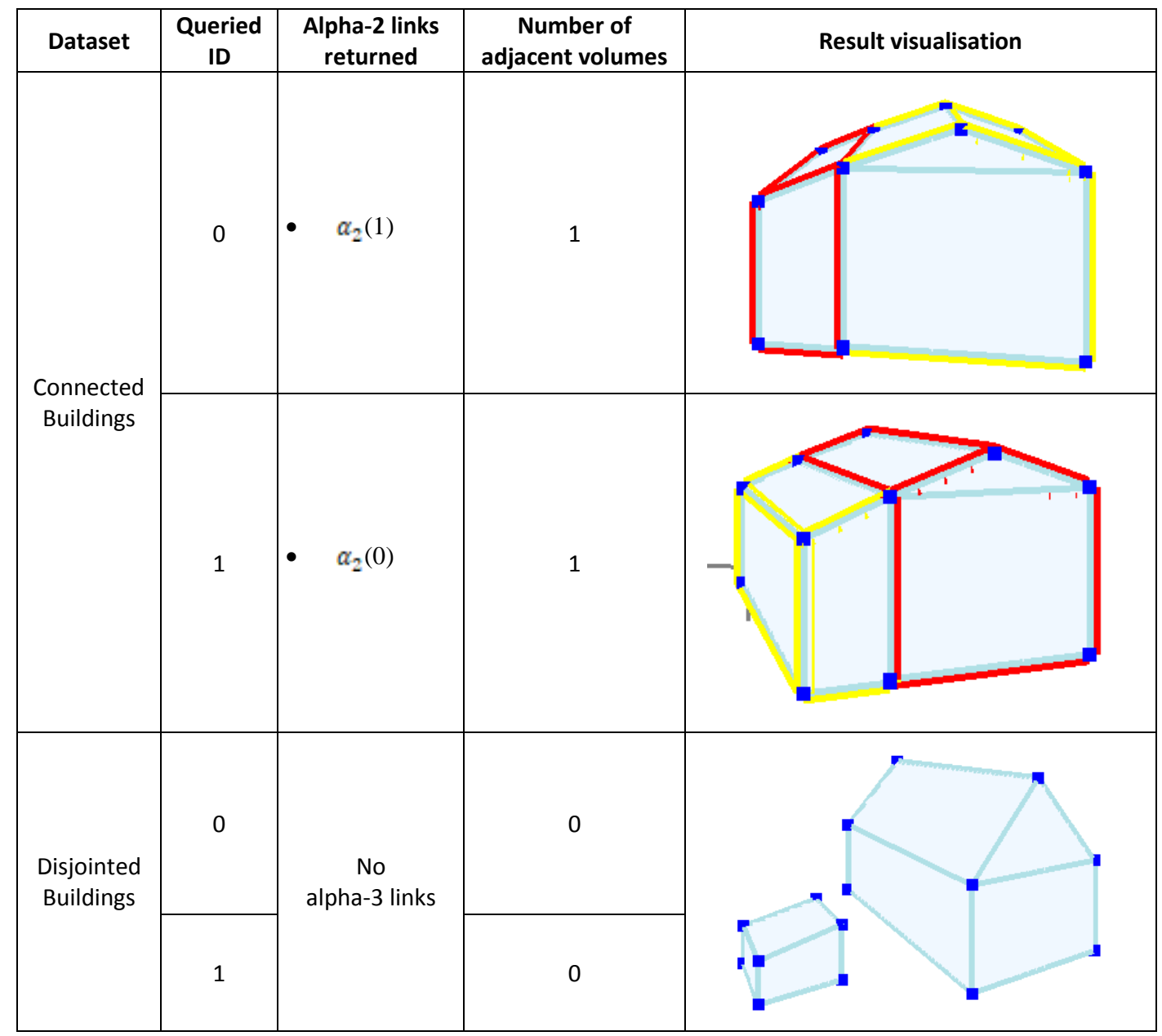

Table 4. Volume-to-Volume adjacency results

\section{CONCLUSION}

In terms of buildings and its elements, the XLinks topology mechanism utilised by CityGML is able to reference two volumes by their common surface or reference surfaces to represent that they belong to the same volume. Nonetheless, the XLinks method of referencing is unable to describe the connectivity between objects or elements and no topological relationships are maintained between objects of different dimensions. In contrast, the CACC topological data structure as a means of providing 3D topology was found to support the preservation of topological primitives in each dimension which results in topological links that maintain connectivity information. The connectivity information provided by the topological links also includes connectivity between objects of different dimensions as any topological link can be decomposed to lower dimensions or vice versa.

The adjacency results presented in this paper was achieved by implementing a 3D topological data structure which supported the comprehensive maintenance of topological primitives and connectivity information. Adjacency information which describes how objects are connected is the basis of more complex spatial analysis such as 3D navigation, nearest neighbour and others. The preservation of topological primitives and connectivity information provided support for basic adjacencies for buildings and their lower dimension elements. Hence, it is crucial for $3 \mathrm{D}$ modelling standards to emphasise and implement a method of maintaining 3D topology within a 3D model. Future studies may explore other topological data structures that can be implemented for maintaining topological information for buildings in CityGML.

\section{ACKNOWLEDGEMENTS}

This research was partially funded by UTM Research University Grant, Vot Q.J130000.3552.05G34 and Vot Q.J130000.3552.06G41. The CityGML datasets were obtained freely from the CityGML Open Data Initiatives.

\section{REFERENCES}

Azri, S., Ujang, U., François, A., Darka, M., Alias, A.R., 2014. Spatial Access Method for Urban Geospatial Database Management: An Efficient Approach of 3D Vector Data Clustering Technique, 9th International Conference on Digital Information Management (ICDIM). IEEE, Bangkok, Thailand. 
Azri, S., François, A., Ujang, U., Darka, M., Alias, A.R., 2015. Crisp Clustering Algorithm for 3D Geospatial Vector Data Quantization, Lecture Notes in Geoinformation and Cartography. Springer Verlag, pp. 71-85.

Azri, S., Ujang, U., Castro, F.A., Abdul Rahman, A., Mioc, D., 2016. Classified and clustered data constellation: An efficient approach of 3D urban data management. ISPRS Journal of Photogrammetry and Remote Sensing 113, 30-42.

Biljecki, F., Ledoux, H., Du, X., Stoter, J., Soon, K. H., \& Khoo, V. (2016). The most common geometric and semantic errors in CityGML datasets. ISPRS Annals of the Photogrammetry, Remote Sensing and Spatial Information Sciences, 4(2W1), 13-22.

Boguslawski, P., Gold, C. M., \& Ledoux, H. (2011). Modelling and analysing 3D buildings with a primal/dual data structure. ISPRS Journal of Photogrammetry and Remote Sensing. 66(2), 188-197.

Bruzzone, E., \& De Floriani, L. (1991). Extracting adjacency relationships from a modular boundary model. Computer-Aided Design, 23(5), 344-355.

Carlsson, G. (2014). Topological pattern recognition for point cloud data. Acta Numerica, 23, 289-368.

Ghawana, T., \& Zlatanova, S. (2012). Increasing Significance of 3D Topology for Modelling of Urban Structures. Geospatial World Forum 2012. 23-27 April. Amsterdam, The Netherlands.

Gröger, G., \& Plümer, L. (2012). CityGML - Interoperable semantic 3D city models. ISPRS Journal of Photogrammetry and Remote Sensing, 71, 12-33.

Keling, N., Mohamad Yusoff, I., Lateh, H., Ujang, U., 2017. Highly Efficient Computer Oriented Octree Data Structure and Neighbours Search in 3D GIS, in: Abdul-Rahman, A. (Ed.), Advances in 3D Geoinformation. Springer International Publishing, Cham, pp. 285-303.

Kolbe, T. H. (2009). Representing and exchanging 3D city models with CityGML. In $3 D$ geo-information sciences (pp. 1531): Springer.

Li, L., Luo, F., Zhu, H., Ying, S., \& Zhao, Z. (2016). A twolevel topological model for 3D features in CityGML. Computers, Environment and Urban Systems, 59, 11-24.

Martinez-Llario, J., Coll, E., Núñez-Andrés, M., \& FemeniaRibera, C. (2017). Rule-based topology system for spatial databases to validate complex geographic datasets. Computers \& Geosciences, 103, 122-132.

Mohd, Z.H., Ujang, U., Choon, T.L., 2017. Heritage House Maintenance using 3D City Model Application Domain Extension Approach. International Archives of the Photogrammetry, Remote Sensing \& Spatial Information Sciences 42.

Open Geospatial Consortium (2012). OGC City Geography Markup Language (CityGML) Encoding Standard. Open Geospatial Consortium.
Rook, M., Biljecki, F., \& Diakité, A. (2016). Towards automatic semantic labelling of 3D city models. ISPRS Annals of the Photogrammetry, Remote Sensing and Spatial Information Sciences, 4(2W1), 23-30.

Salleh, S., Ujang, U., Azri, S., \& Choon, T. L. (2018). Cell Complexes Topological Links for Buildings in CityGML. Int. Arch. Photogramm. Remote Sens. Spatial Inf. Sci., XLII-4/W10, 165-169.

Ujang, U., Abdul Rahman, A., Anton, F. (2014). Threedimensional Geospatial Compact Abstract Cell Complexes Data Structure for Urban Air Pollution Modelling. Universiti Teknologi Malaysia.

Ujang, U., Anton Castro, F., \& Azri, S. (2019). Abstract Topological Data Structure for 3D Spatial Objects. ISPRS International Journal of Geo-Information, 8(3), 102.

Ujang, U., Azri, S., Zahir, M., Abdul Rahman, A., Choon, T.L., 2018. Urban Heat Island Micro-Mapping via 3D City Model. Int. Arch. Photogramm. Remote Sens. Spatial Inf. Sci. XLII4/W10, 201-207.

Worboys, M. F., \& Duckham, M. (2004). GIS: A Computing Perspective. CRC press.

Revised August 2019 